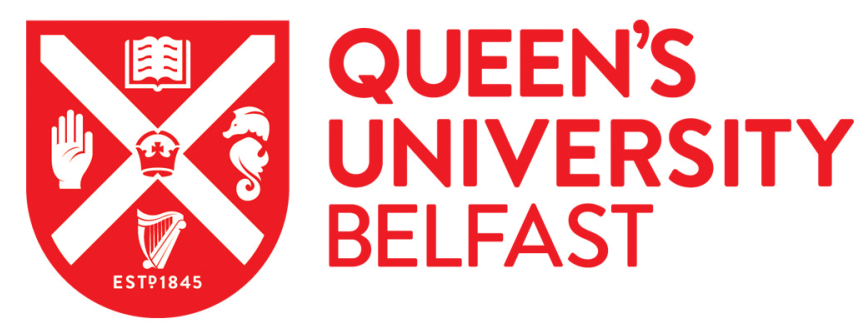

\title{
Spectral Sequences of Type la Supernovae. I. Connecting Normal and Subluminous SNe la and the Presence of Unburned Carbon
}

Heringer, E., Van Kerkwijk, M. H., Sim, S. A., \& Kerzendorf, W. E. (2017). Spectral Sequences of Type la Supernovae. I. Connecting Normal and Subluminous SNe la and the Presence of Unburned Carbon. The Astrophysical Journal, 846(1), 15. https://doi.org/10.3847/1538-4357/aa8309

\section{Published in:}

The Astrophysical Journal

Document Version:

Publisher's PDF, also known as Version of record

Queen's University Belfast - Research Portal:

Link to publication record in Queen's University Belfast Research Portal

\section{Publisher rights}

(C) Copyright 2017 IOP Publishing. This work is made available online in accordance with the publisher's policies. Please refer to any applicable terms of use of the publisher.

\section{General rights}

Copyright for the publications made accessible via the Queen's University Belfast Research Portal is retained by the author(s) and / or other copyright owners and it is a condition of accessing these publications that users recognise and abide by the legal requirements associated with these rights.

Take down policy

The Research Portal is Queen's institutional repository that provides access to Queen's research output. Every effort has been made to ensure that content in the Research Portal does not infringe any person's rights, or applicable UK laws. If you discover content in the Research Portal that you believe breaches copyright or violates any law, please contact openaccess@qub.ac.uk. 


\title{
Spectral Sequences of Type Ia Supernovae. I. Connecting Normal and Subluminous SNe Ia and the Presence of Unburned Carbon
}

\author{
E. Heringer ${ }^{1}$ (1) , M. H. van Kerkwijk ${ }^{1}$ (i), S. A. $\operatorname{Sim}^{2}$ (i), and W. E. Kerzendorf ${ }^{3}$ (1) \\ ${ }^{1}$ Department of Astronomy \& Astrophysics, University of Toronto, 50 Saint George Street, Toronto, ON M5S 3H4, Canada \\ 2 Astrophysics Research Centre, School of Mathematics and Physics, Queens University Belfast, Belfast BT7 1NN, UK \\ European Southern Observatory (ESO), Karl-Schwarzschild-Straße 2, D-85748 Garching, Germany \\ Received 2017 June 29; revised 2017 July 26; accepted 2017 July 26; published 2017 August 28
}

\begin{abstract}
Type Ia supernovae (SNe Ia) are generally agreed to arise from thermonuclear explosions of carbon-oxygen white dwarfs. The actual path to explosion, however, remains elusive, with numerous plausible parent systems and explosion mechanisms suggested. Observationally, SNe Ia have multiple subclasses, distinguished by their light curves and spectra. This raises the question of whether these indicate that multiple mechanisms occur in nature or that explosions have a large but continuous range of physical properties. We revisit the idea that normal and $91 \mathrm{bg}-$ like SNe can be understood as part of a spectral sequence in which changes in temperature dominate. Specifically, we find that a single ejecta structure is sufficient to provide reasonable fits of both the normal SN Ia SN $2011 \mathrm{fe}$ and the 91bg-like SN 2005bl, provided that the luminosity and thus temperature of the ejecta are adjusted appropriately. This suggests that the outer layers of the ejecta are similar, thus providing some support for a common explosion mechanism. Our spectral sequence also helps to shed light on the conditions under which carbon can be detected in premaximum SN Ia spectra-we find that emission from iron can "fill in" the carbon trough in cool SNe Ia. This may indicate that the outer layers of the ejecta of events in which carbon is detected are relatively metal-poor compared to events in which carbon is not detected.
\end{abstract}

Key words: supernovae: general - supernovae: individual (SN 2011fe, SN 2005bl)

Supporting material: figure set

\section{Introduction}

While most type Ia supernovae (SNe Ia) are remarkably uniform, it has long been known that there are subclasses whose events share distinguishing features, such as the overluminous "91T-like" and subluminous "91bg-like" (Filippenko 1997). The number of subclasses has grown with the number of available spectra, with recent reviews distinguishing almost 10 (Chomiuk et al. 2016; Taubenberger 2017).

For many of the subclasses, there are associated proposed evolutionary channels and physical explosion mechanisms. Only a few, however, have a consensus on which one is correct. Furthermore, just as SN types II, IIb, Ib, and Ic have turned out to reflect not different physical origins but rather a sequence in the amount of stellar envelope stripped before core collapse, some of the observed Ia subtypes may reflect variations in explosion properties rather than different explosion mechanisms.

Nugent et al. (1995) was among the first to propose that SNe Ia belonging to different subclasses could originate from a spectral sequence in luminosity. This parameter sets the temperature profile of the ejecta, determining the ionization state of the elements therein and thus shaping the observed spectral features. In this scenario, the amount of ${ }^{56} \mathrm{Ni}$ synthesized in the explosion would be the primary factor driving the sequence. A similar approach was employed by Hachinger et al. (2008) to investigate in detail the strength of silicon features.

Indeed, observationally, some subclasses seem to form continuous sequences. For instance, Branch et al. (2006) noted that their four subclasses, which are based on the pseudoequivalent widths of the $\lambda 6355$ and $\lambda 5972 \mathrm{Si}$ II absorption features, show a continuous distribution (see Figure 3 below).
Similarly, Benetti et al. (2005) and Sasdelli et al. (2015) showed that by using different techniques (the velocity gradient of the $\lambda 6355 \mathrm{Si}$ II line and principle-component analysis, respectively), the known subgroups can be recovered but are not strongly disjointed, with some objects classified as "transitional" types.

Here, we focus specifically on the normal and 91bg-like subclasses. Compared to normal $\mathrm{SNe}$, 91bg-like events are fainter, decline faster, have a "titanium trough" near $4200 \AA$, and show stronger Si II $\lambda 5972$ and O I $\lambda 7774$ lines in their spectra (Doull \& Baron 2011; Taubenberger 2017). Moreover, the velocity inferred from the Si II $\lambda 6355$ line evolves more rapidly and on average settles at lower values in the postmaximum spectra (Benetti et al. 2005).

Also for normal and 91bg-like SNe Ia, it is currently unclear whether they share the same progenitor scenario or arise from different channels. For instance, Sasdelli et al. (2017) tried to classify explosion mechanisms based on their predicted spectra and found that, while all mechanisms had shortcomings, equalmass violent mergers fit 91bg-like SNe Ia quite well, while detonations of sub-Chandrasekhar white dwarfs and delayed detonations better represented normal SNe Ia. From the distribution of inferred ${ }^{56} \mathrm{Ni}$ masses, Piro et al. (2014) suggested that normal and 91bg-like SNe Ia might arise from the double-detonation (Livne 1990) and direct-collision (Dong et al. 2015) scenarios, respectively. In contrast, e.g., Graur et al. (2017) suggested a common origin on statistical grounds: while $91 \mathrm{bg}$-like SNe Ia are preferentially found in massive galaxies, they seem to "cut into the share" of the normal SNe Ia.

We focus on the spectroscopic differences between two very well studied SNe that are thought to be representative of their subclasses: the normal SN 2011fe and 91bg-like SN 2005bl. 
For both, using the technique known as "abundance tomography" (e.g., Stehle et al. 2005), the observed spectral evolution has been used to probe different layers of the ejecta, with deeper ones exposed as the photosphere recedes. For SN 2011fe, Mazzali et al. (2014) concluded that the progenitor likely had subsolar metallicity and that the density profile of the ejecta was between the prediction from the benchmark W7 model of Nomoto et al. (1984) and the WDD1 delayeddetonation model of Iwamoto et al. (1999). For SN 2005bl, Hachinger et al. (2009) first optimized their synthetic spectra assuming a W7 density profile and then used energy and mass scalings to improve their fits, finding the best agreement when the original mass $\left(1.38 M_{\odot}\right)$ was maintained but the kinetic energy was reduced by $30 \%$.

Thus, beyond differences in explosion energy, the two analyses yielded somewhat different density profiles. Furthermore, the abundances also differ. For instance, for titanium, the element responsible for the $4200 \AA$ titanium trough distinctive of 91bg-like supernovae, SN 2011fe yielded a total mass $>10$ times higher than SN 2005bl in the outer layers $\left(v \gtrsim 7,800 \mathrm{~km} \mathrm{~s}^{-1}\right)$, whereas SN 2005bl contains more titanium in the inner layers.

A known issue with tomography is the presence of strong degeneracies; i.e., multiple sets of parameters may give good agreement. Hence, in this first paper in a series in which we hope to investigate the observational constraints on the explosions with tomography, our primary goal is to determine whether, even though the density and abundance profiles found for both supernovae were different, the spectral differences can, in fact, be understood from variations in just one or a few parameters. We also report on a by-product of our investigation, which is that the carbon feature is visible only along a limited range in our spectral sequences; this may shed light on why carbon is only sporadically detected in SNe Ia ( $~ 30 \%$ of events with early enough spectra; e.g., Folatelli et al. 2012).

\section{Methodology}

To explore the physical properties that distinguish 91bg-like from normal SNe Ia, we use the spectral synthesis code TARDIS (Kerzendorf \& Sim 2014). TARDIS is a Monte Carlo code that is very similar to the code used to analyze SN $2011 \mathrm{fe}$ and SN 2005bl (Mazzali 2000), and hence our approach is to start from the available tomography (Hachinger et al. 2009; Mazzali et al. 2014). After verifying that we reproduced the earlier results, our goal is then to see for each object if we can make the synthetic spectra approach the observed spectra of the other object through a low-dimensional sequence in physical properties.

Here, for our first attempt, we follow Nugent et al. (1995) and Hachinger et al. (2008) and search for a sequence based on explosion brightness. We extend previous work by analyzing spectra taken not just near maximum light but also before and after and by investigating the possibility of a sequence in the titanium mass fraction $X(\mathrm{Ti})$.

Specifically, for our simulations, we used TARDIS v1.5dev2685, which calculates a synthetic spectrum for given density and abundance profiles, an observed brightness, the time since the explosion, and the position of the pseudophotosphere (i.e., below which the ejecta are optically thick). In TARDIS, the ejecta are assumed to be in homologous expansion with parameters given in velocity space, and the modeled photon interactions are absorption and emission by lines (in the Sobolev approximation) and electron scattering (for details, see Kerzendorf \& Sim 2014).

We adopt TARDIS settings similar to those in previous works; see the Appendix and Kerzendorf \& Sim (2014) for a detailed explanation. For our "default" ejecta structure of SN $2011 \mathrm{fe}$, we use the data available in Table 6 and Figure 10 of Mazzali et al. (2014), and, for SN 2005bl, we use the same input parameters as Hachinger et al. (2009) for their best model "05bl-w7e0.7," as in their Table A1. Starting with these, we then scale the luminosity of the explosion, following what was done by Hachinger et al. (2008) to investigate the strength of the Si II $\lambda 6355$ and $\lambda 5972$ lines but focusing on the spectral sequence itself, not restraining the analysis to near-maximum spectra, and varying the titanium abundance.

For comparison purposes, all the spectra shown in this paper are normalized by the mean flux over a wavelength window of 4000-9000 А.

\section{Results}

We compare our models with observed spectra ${ }^{4}$ for three epochs in Figure 1 (sets a-f). One sees that there is good agreement with the default models, showing that TARDIS allows one to reproduce the previous results, although the agreement is worse at late times, especially for SN 2005bl (see Section 5). In detail, also near and premaximum, our synthetic spectra are not entirely consistent with those previously published. This may reflect differences between TARDIS and the Mazzali (2000) code, small differences in the (interpretation of the) density and abundance profiles of SN 2011fe, and the choice of atomic data. Nevertheless, the differences between the codes are no larger than those between the simulated and observed spectra, giving us confidence that relative changes are reliable.

\subsection{Luminosity Scaling}

In Figure 1, we show simulated spectral sequences in which the luminosity of the SN 2011fe and SN 2005bl models are scaled down and up, respectively, toward the luminosity of the other supernova. One sees that the observed SN 2005bl spectra are quite well reproduced by some of the fainter SN $2011 \mathrm{fe}$ model spectra (sets a-c.)

Indeed, our cooler SN 2011fe models not only approach the overall spectral shape of SN $2005 \mathrm{bl}$ but also succeed in reproducing some of the general spectroscopic features of 91bg-like SNe Ia (see Section 1)_for instance, the formation of the titanium trough and the stronger features due to the Si II $\lambda 5972$ and O I $\lambda 7774$ lines. This is accomplished without enhancing the abundance of these elements and is simply an effect of the changes in ionization states.

The above corroborates the idea that luminosity is a determining factor for the spectral differences. Looking in more detail, we see that the luminosity scale factor of 0.25 for SN 2011fe that produces a good match to SN 2005bl near maximum corresponds to a brightness similar to the observed one for the latter. However, the same is not true for the premaximum and postmaximum models (sets a and c), where the best match is for a luminosity ratio of 0.33 , while the observed ratio is about 0.18 .

\footnotetext{
4 Reduced spectra were retrieved from the WISeREP archive (Yaron \& Gal-Yam 2012) at https://wiserep.weizmann.ac.il/. For details of the data reduction, see Mazzali et al. (2014) and Taubenberger et al. (2008).
} 

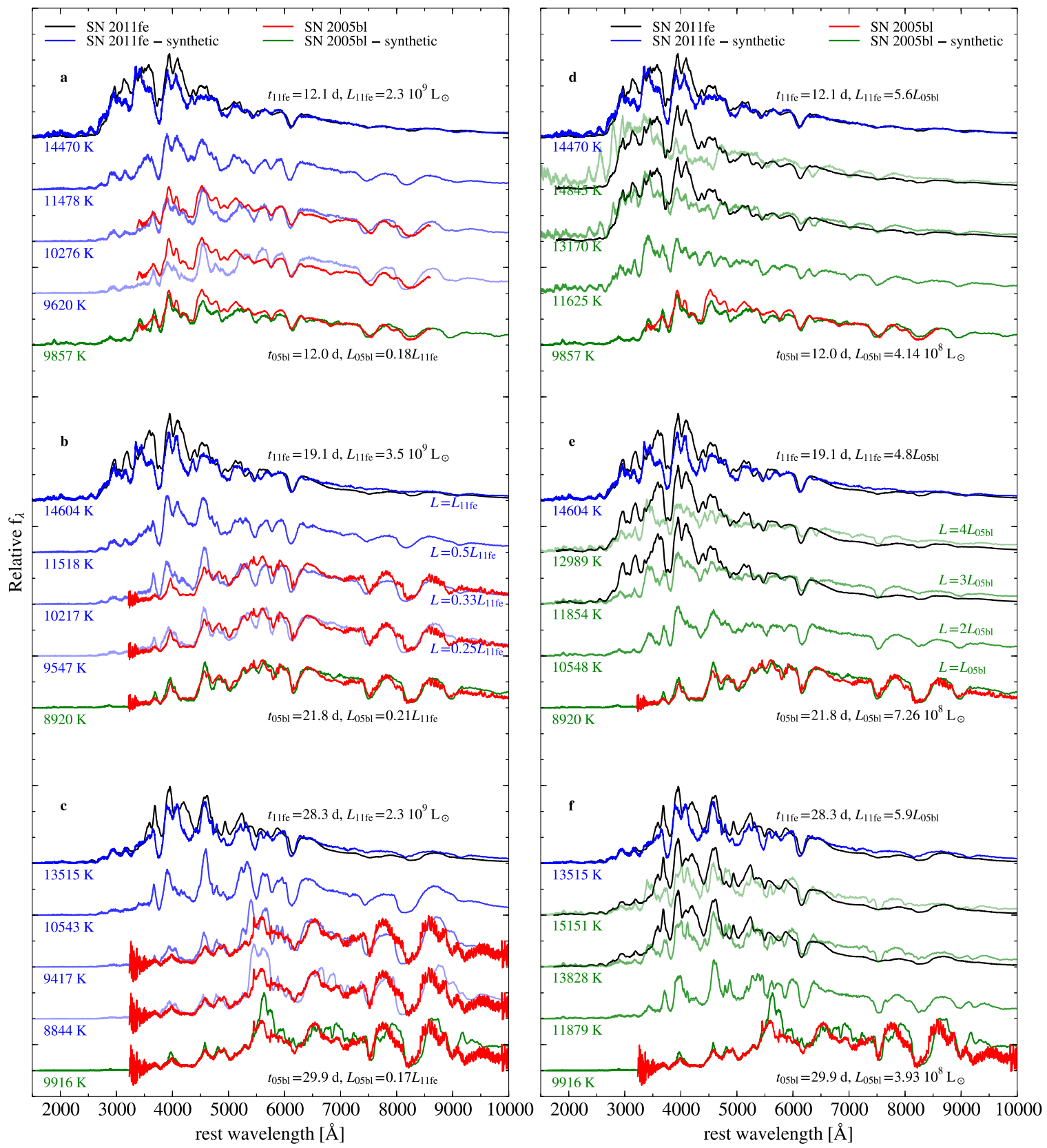

Figure 1. Comparison between spectra of the normal SN 2011fe and the 91bg-like SN 2005bl with models at three different epochs. In both panels and in each set, the observed spectra for SN 2011fe and SN 2005bl are shown as black and red lines, respectively, and synthetic spectra based on literature tomography are shown as blue (Mazzali et al. 2014) and green (Hachinger et al. 2009) lines, respectively. Also shown are synthetic spectra obtained with increasing changes in luminosity and in increasingly fainter lines using the best-fit models for SN 2011fe (left panel) and SN 2005bl (right panel). One sees that the SN 2011fe model can reproduce the observed spectra of SN 2005bl at lower luminosity, and, similarly, the SN 2005bl model can reproduce the observed spectra of SN $2011 \mathrm{fe}$ at higher luminosity.

(The complete figure set ( 2 images) is available.)

In contrast, looking at the inner temperatures $\left(T_{\text {inner }}\right.$; see Figure 1) associated with the models, we find that, near maximum, the $0.25 L_{11 f e}$ model is closest in temperature to the default SN $2005 \mathrm{bl} \mathrm{model}\left(T_{\text {inner }}=9547 \mathrm{~K}\right.$ versus $\left.8920 \mathrm{~K}\right)$. More importantly, the temperature profile of the ejecta across the lineforming regions is similar between the scaled models for SN 2011fe and SN 2005bl. This is relevant because the precise inner temperature can vary significantly $( \pm 1000 \mathrm{~K})$ depending on the adopted inner radius and still produce consistent spectra. This is because the effective radius at which photons originate and the temperature at that radius are what determine the luminosity and the spectrum, and those may not be affected by the precise location of the inner boundary. This suggests the idea that rather than comparing SNe at the same phase or brightness, one ought to compare the spectra produced when the ejecta are at a similar state with a similar temperature profile. To also match the luminosity, one then may have to take into account the difference in rise time ( 17 and 19 days for SN 2005bl and SN 2011fe, respectively; Taubenberger et al. 2008; Mazzali et al. 2014) and thus correct for the timescale on which the SN evolves (see Section 5.1).

While it was previously known that the temperature is important (e.g., Nugent et al. 1995; Mazzali et al. 1997; Branch 
et al. 2006; Blondin et al. 2013), the fact that a sequence of spectra produced from a common ejecta structure can produce both subclasses of SNe Ia by a change in temperature lends support to the idea that their outer ejecta are quite similar, despite degeneracies in, for example, the chosen density profile.

Consistent with the dominance of temperature in determining the spectral sequence is that not only do the fainter synthetic spectra based on SN 2011 fe resemble SN 2005bl, the reverse is true as well: the brighter synthetic spectra based on the SN 2005bl model qualitatively resemble those of normal $\mathrm{SNe}$ - in particular, there is no clear $4200 \AA$ Ti trough. In more detail, however, the cooler SN 2011 fe models (sets a-c) seem to exhibit a better agreement with the observed spectra of SN 2005bl than the hotter models of SN 2005bl do when compared to SN 2011fe observations (sets d-f). This suggests that the density profile found for SN 2011 fe might be closer to a common density profile for SNe Ia than the energy-scaled W7 density profile employed for the SN 2005bl simulations.

Our cooler SN 2011fe models not only approach the overall spectral shape of SN $2005 \mathrm{bl}$ but also succeed in reproducing some of the general spectroscopic features of 91bg-like SNe Ia (see Section 1)-for instance, the formation of the titanium trough and the stronger features due to the Si II $\lambda 5972$ and O I $\lambda 7774$ lines. This is accomplished without enhancing the abundance of these elements and is simply an effect of the changes in ionization states.

\subsection{Luminosity and Titanium Sequences}

In Figure 2, we start with the default synthetic spectrum of SN 2011fe at maximum to explore how the spectra are affected in more detail by both the luminosity and thus temperature (left panel) and the titanium abundance (right panel). As a function of luminosity, one sees that the $4200 \AA$ titanium trough becomes progressively clearer as the luminosity decreases below $\sim 0.27 L_{11 \text { fe }}$ and gets blended with the $\mathrm{Si}$ II feature near $4000 \AA$. Other spectral features also change continuously, with the Ca II near-IR triplet feature near $8300 \AA$ becoming stronger as the luminosity decreases (as fewer calcium atoms are more than singly ionized) and the Si II feature near $6100 \AA$ first becoming stronger (for a similar reason) and then starting to blend with other features for $L \lesssim 0.24 L_{11 \mathrm{fe}}$.

We scaled the titanium and chromium mass fractions throughout the ejecta by a factor of 0-20 while imposing the condition that $X(\mathrm{Ti})=X(\mathrm{Cr})$, as in Stehle et al. (2005). Both elements are adjusted at the expense of the most abundant element in each layer (for which this is a small change: the default model contains only $\sim 3 \times 10^{-3} M_{\odot}$ of titanium above the photosphere). We use two luminosities, 1.0 and $0.25 L_{11 \text { fe, } \max }$, chosen such that we can investigate both how much titanium a typical normal SN Ia could "hide" at maximum without developing a Ti trough and by what amount we can decrease the titanium in cooler spectra without losing the trough.

From Figure 2, one sees that the amount of Ti in the ejecta will strongly influence the shape of the underlying continuum and thus the color. This effect holds for both luminosities and is as expected, since titanium and chromium, like other iron-group elements, have many transitions in the blue and UV range and thus efficiently block UV/blue light (Kromer et al. 2010). For the spectra with $L=L_{11 \mathrm{fe} \text {,max }}$ (top set), the shapes of the spectral features remain nearly unchanged, except for the $\mathrm{Ca}$ II $\mathrm{H}$ and $\mathrm{K}$ feature near $3700 \AA$. At $L=0.25 L_{11 \mathrm{fe}, \max }$ (bottom set), the photosphere is cooler ( 9500 versus $14,500 \mathrm{~K}$ ), and the changes in color are more drastic. The Ti trough near $4200 \AA$ is conspicuous as long as $X(\mathrm{Ti})$ is larger than 0.05 times the default, and it disappears when $\mathrm{Ti}$ (and $\mathrm{Cr}$ ) are absent from the ejecta. For large amounts of $\mathrm{Ti}$ at factors $>5$ above default, the relative depth of the trough diminishes as the spectra become redder. Overall, like Blondin et al. (2013), we conclude that the $4200 \AA$ trough primarily reflects the degree of ionization of titanium.

\subsection{Carbon Features}

Taubenberger et al. (2008) reported the likely detection of unburned carbon in the early spectra of SN 2005bl due to the C II $\lambda 6580$ transition (seen next to the red shoulder of the silicon feature near $6100 \AA$ ). While this feature is not restrained to $91 \mathrm{bg}$-like SNe Ia (Blondin et al. 2012), we note that our scaled model for SN 2011 fe fails to reproduce it (see set a). Interestingly, this feature is present in our near-maximum synthetic spectra (set b), but only for $L=0.50 L_{11 \text { fe,max }}$. This might shed light on why carbon is only sporadically detected in the early spectra of SNe Ia. In particular, the spectral series in Figure 2 reveals that the carbon signature is only clearly present in the luminosity range of $0.42 L_{11 \mathrm{fe}, \max } \lesssim L \lesssim 0.98 L_{11 \mathrm{fe} \text {, max }}$.

Analyzing the spectral energy distribution according to each element in our spectra, we find that the presence of the carbon trough arises from a balance between iron emission "filling in" the carbon trough in the cooler spectra and not enough carbon being singly ionized in the hottest cases. This finding could explain the correlation that events in which carbon is detected tend to exhibit bluer optical/near-UV colors than their counterparts in which it is not (Thomas et al. 2011; Folatelli et al. 2012; Milne \& Brown 2012; Silverman \& Filippenko 2012). Since iron-group elements effectively redistribute UV light to longer wavelengths, blue continua and carbon features could both be signatures of relatively low metal content (Lentz et al. 2000; Walker et al. 2012; but see Sauer et al. 2008). We discuss this further in Section 5.2.

\subsection{The Strengths of the Si II $\lambda 6355$ and $\lambda 5972$ Features}

To put the effects of changes in luminosity and temperature in a larger context, we investigate how they affect features that have been used to classify SNe Ia. Specifically, we use the strong features near 6100 and $5700 \AA$-due to Si II $\lambda 6355$ and $\lambda 5972$ - that define the core-normal, shallow-silicon, cool, and broad-line subclasses of Branch et al. (2006, 2009). Here, the cool subclass overlaps with the faint group defined by Benetti et al. (2005), which we generically call 91bg-like. For each spectrum shown in the left panel of Figure 2, we compute pseudo-equivalent widths ( $\mathrm{pEW}$ ) following the prescription of Silverman et al. (2012b). We estimate uncertainties following Liu et al. (2016) using a Monte Carlo routine to generate mock spectra with noise properties like the data (estimated by smoothing the spectrum and computing the rms between the smoothed spectrum and the data in a wavelength window of $40 \AA$ ). By analyzing multiple TARDIS runs with identical inputs, we find that the resulting uncertainties slightly underestimate the true ones by $20 \%$ (for which we correct).

We remeasured pEWs of objects from the Berkeley Supernova Ia Program (BSNIP; Silverman et al. 2012a) and show them in Figure 3 (which can be compared with Figure 10 from Silverman et al. 2012b; our pEW are consistent). Overlaid are the pEWs we measured from the synthetic spectra based on the SN $2011 \mathrm{fe}$ model at maximum, but with scaled luminosity. One sees that the spectral sequence crosses through all four subclasses displayed. 

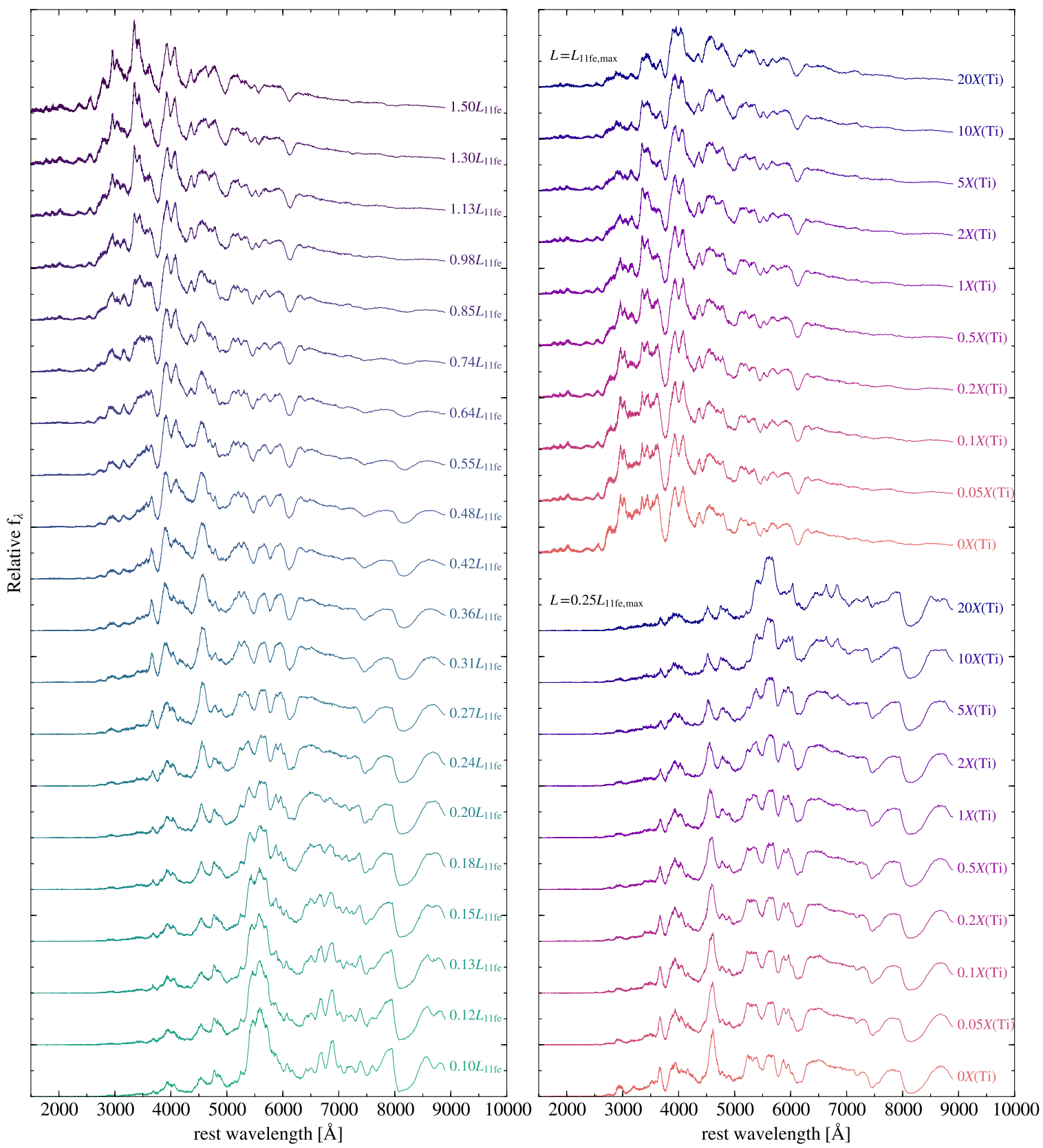

Figure 2. Simulated spectra for a range in luminosity and titanium abundance. Left panel: varying the luminosity by a factor of $0.1-1.5$ in the model that best fits the maximum-light spectrum of SN $2011 \mathrm{fe}$. Note how the titanium trough near $4200 \AA \AA$ develops over a relatively small range in luminosity, starting to become clear at $\sim 0.36 L_{11 \text { fe }}$ and being fully formed at $\sim 0.27 L_{11 \text { fe. }}$. The trough becomes less obvious at very low luminosity, where line blanketing suppresses the whole blue part of the spectrum. Right panel: varying the titanium (and chromium; see text) abundance by a factor of $0-20$ for both the best model of SN $2011 \mathrm{fe}$ (top set) and a model in which the luminosity is four times lower. One sees that the spectra are much more sensitive to the titanium abundance at lower luminosity.

(The complete figure set (2 images) is available.)

The brightest explosions are in the shallow-silicon region, reflecting that most of the silicon is more than singly ionized at the corresponding high temperatures. Toward lower brightness, the fraction of singly ionized Si increases strongly (while only a negligible fraction is neutral); the Si II $\lambda 6355$ feature strengthens until it saturates near a value of $\sim 130 \AA$, while the Si II $\lambda 5972$ feature becomes progressively stronger (Hachinger et al. 2008).

\section{Modeling Uncertainties}

An important limitation of our analysis follows from the approximation of the incoming radiation field from the pseudo-photosphere as blackbody radiation. This approximation becomes progressively worse starting about a week after maximum, as the ejecta evolve and the photosphere moves in too far. For comparison with previous work, we nevertheless included some simulations at such late epochs.

It is generally suggested that the macroatom TARDIS mode for line treatment is used instead of the downbranch mode that we employ, since the former provides a more physical approximation. Specifically, the macroatom approximation better describes de-excitation cascades (and multiplephoton excitation, though those are less likely to be important) than does the simpler downbranch mode. Nevertheless, we 


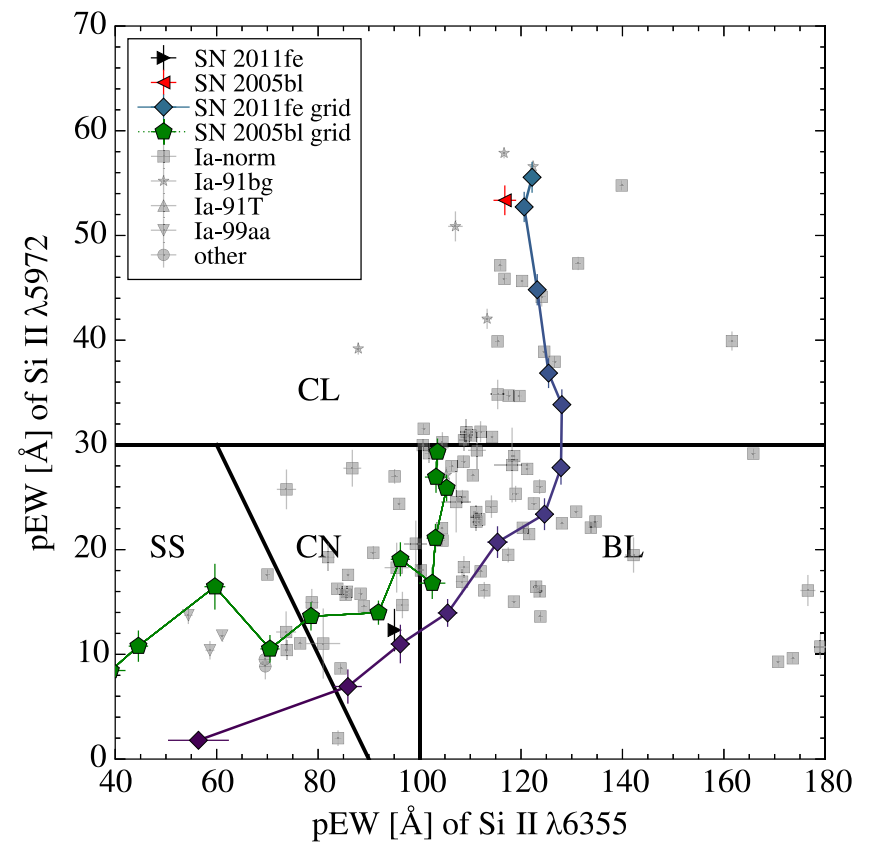

Figure 3. Observed and simulated pEWs of the Si II $\lambda 6355$ and $\lambda 5972$ features (after Figure 10 of Silverman et al. 2012b). The four regions indicate the four subclasses identified by Branch et al. (2009): core-normal (CN), shallowsilicon (SS), cool (CL), and broad-line (BL). Observed widths (gray points) are for objects from the BSNIP survey (Silverman et al. 2012a), and simulated widths are for spectra based on the best-fit model for SN $2011 \mathrm{fe}$ at maximum but with the luminosity scaled by a factor of $0.31-1.5$ (connected diamonds, color-coded as in the left panel of Figure 2) and for SN 2005bl scaled by a factor of 1.13-7.2 (green pentagons). For even lower luminosities, the features blend, making it difficult to measure the pEWs.

(The complete figure set (2 images) is available.)

retain the downbranch approximation for consistency with the earlier abundance tomography models on which our study is based; to consistently use the macroatom mode would require redoing the tomography of SN 2011fe and SN 2005bl, which is beyond the scope of this work.

However, while Kerzendorf \& Sim (2014) found few substantial differences between macroatom and downbranch calculations for the model they considered, our particular model is noticeably affected by this choice. In Figure 4, we show synthetic spectra for the "default" model for the early spectra of SN 2011fe and SN 2005bl (set a in Figure 1), computed using both the macroatom and downbranch modes. Looking at the simulations in detail, we find that the emission and absorption of $\mathrm{Si}$ and $\mathrm{Mg}$ in particular differ substantially between the two treatments, with cooler spectra more strongly affected. Clearly, this is important for detailed fits, and we hope to investigate the origin of this difference in the future.

To help give a sense of the differences, we have added an extra version of all of the figures in the electronic version of this paper, in which the synthetic spectra are computed with the macroatom line interaction mode. While our main conclusions are not affected by these differences, the comparison between SN 2005bl and a "fainter" version of SN 2011fe are less strikingly similar. Of course, for these simulations, the starting models are not optimal, since the tomography was done using a different treatment. It will require tomography using the macroatom mode, however, to test whether starting from proper initial conditions would make the spectra look more similar again.

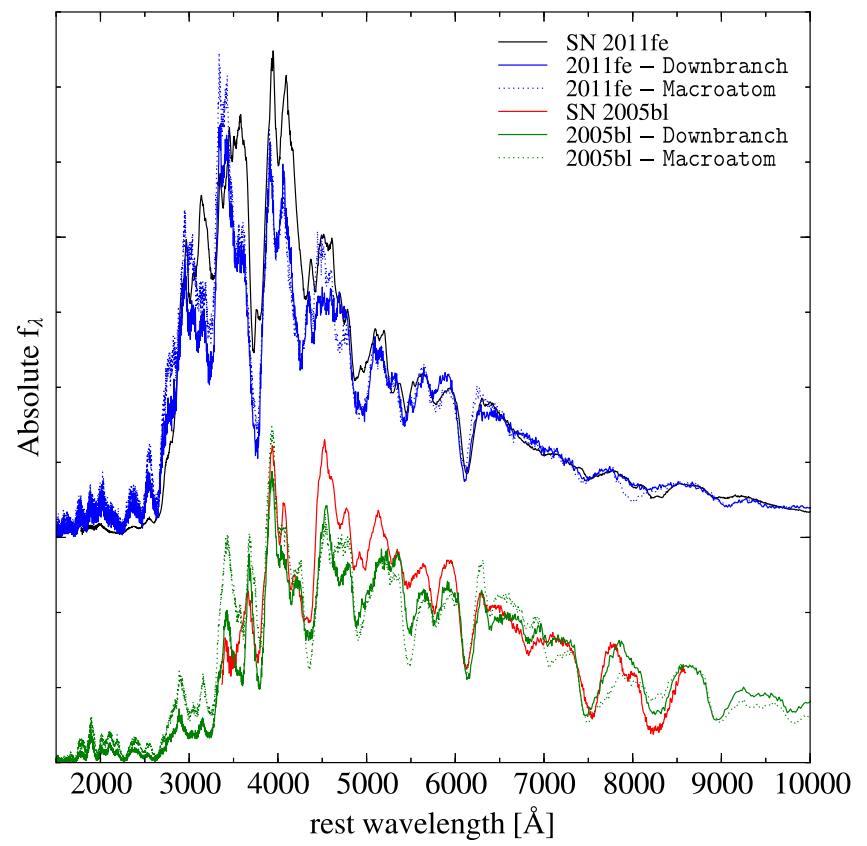

Figure 4. Comparison between synthetic spectra computed using the downbranch (solid lines) and macroatom (dashed lines) line interaction modes. For reference, the observed spectra of SN 2011fe (black line) and SN 2005bl (green lines)are also shown, as in Figure 1, set a.

\section{Conclusions}

\subsection{Implications for the Normal and 91bg-like Subtypes}

We have shown that for the same ejecta structure, synthetic spectra can reproduce observations of both the normal SN Ia SN 2011fe and the 91bg-like SN 2005bl, with only the input luminosity adjusted such that the temperatures in the ejecta are similar to those found in earlier fits in the literature. In particular, the titanium trough and the evolution of the strength of the silicon, oxygen, and calcium features are in agreement with the data. The luminosities themselves are not always consistent with the observed ones. One would likely obtain better agreement with the observed spectra at the correct luminosity if one were to take into account that SN 2005bl evolved substantially faster than SN $2011 \mathrm{fe}\left(\Delta m_{15,05 \mathrm{bl}}=\right.$ $1.93 \pm 0.10$ mag (Taubenberger et al. 2008) versus $\Delta m_{15,11 \mathrm{fe}}=1.07 \pm 0.06 \mathrm{mag}$ (McClelland et al. 2013)). From initial experiments, we find that there is a degeneracy between the time since explosion and the luminosity; i.e., by reducing the value of time since explosion in the calculations for the scaled-down SN 2011fe model, we can approach the temperature structure and thus the SN 2005bl spectrum similarly well for the correct luminosity.

We have also investigated the effects of changing the titanium and chromium mass fractions, finding that the spectra are relatively insensitive to these. Starting with the SN 2011fe model at maximum, abundance changes by a factor of $0.05-20$ influence the color but do not much affect the shape of the $\mathrm{Ti}$ trough. At lower luminosity, a Ti trough is a generic feature and will exist even for 20 times lower than the nominal abundance of $\sim 3 \times 10^{-3} M_{\odot}$ above the photosphere (Mazzali et al. 2014). Overall, we conclude that normal SNe Ia do not have to be Ti-poor compared to their $91 \mathrm{bg}$-like counterparts but instead simply hotter, causing titanium and chromium to be doubly ionized, thus preventing the formation of the Ti trough (as also found by Blondin et al. 2013.) 
Our results seem suggestive of a common explosion mechanism for $91 \mathrm{bg}$-like and normal $\mathrm{SNe} \mathrm{Ia}$, with the subclasses arising from a continuous set of parameters within a single mechanism. Of course, it does not directly distinguish between mechanisms: indeed, both have been reproduced by delayeddetonation (Blondin et al. 2013), violent-merger (Pakmor et al. 2010, 2012), and double-detonation (Shen et al. 2017) models.

A remaining caveat is that, since the spectra of SN 2005bl can be reproduced by models with distinct ejecta profiles, we cannot truly determine whether its ejecta structure is similar to that of SN 2011fe; in the end, it is mostly an appeal to Occam's razor. Similarly, our results do not exclude potential subdivisions within each of the subclasses. For instance, Dhawan et al. (2017) used the epoch of maximum $B-V$ (whether one or two peaks are seen in the near-infrared) and the epoch of the first near-infrared peak relative to that in the blue to argue that there are two distinct groups of $91 \mathrm{bg}$-like $\mathrm{SNe}$ Ia, of which one exhibits a continuous set of properties with normal $\mathrm{SNe}$ Ia and the other does not. SN 2005bl was part of their sample and was classified as belonging to the second, unconnected group, although the lack of some of the photometric evidence made the classification tentative.

Fortunately, the similarity can be tested: e.g., if indeed $91 \mathrm{bg}$ like SNe Ia have ejecta structures similar to those of normal $\mathrm{SNe} \mathrm{Ia}$, it is possible to predict how their early spectra-which have not yet been observed-will look. Furthermore, with updated simulations, it should be possible to extend the comparison to later times (e.g., Mazzali et al. 2015; Botyánszki \& Kasen 2017), when the inner parts of the ejecta become visible, which surely should be different: 91bg-like SNe Ia must necessarily produce less ${ }^{56} \mathrm{Ni}$.

More generally, our investigation could be extended to other SN Ia subtypes, such as the overluminous 91T-like objects, as well as to objects classified as "transitional" between normal and 91bg-like SNe Ia, such as SN 1986G (Ashall et al. 2016) and SN 2004eo (Pastorello et al. 2007; Mazzali et al. 2008). At the same time, Figure 3 shows that observed SNe Ia do not follow a one-parameter family; it will be interesting to explore what other properties need to be changed to reproduce the SNe Ia more distant from our models.

\subsection{Metal Content in the Outer Layers}

Our cooler models indicate that the detection of the carbon feature near $6100 \AA$ might be hindered by emission from iron, even at premaximum, where it had not hitherto been considered (cf. Branch et al. 2008; Parrent et al. 2012). This suggests that the carbon trough might preferentially be seen in lowmetallicity progenitors or strongly stratified objects, where no or very little of the iron-group elements formed deeper in the ejecta are mixed up to the outer layers.

It is worth noting that $91 \mathrm{bg}$-like $\mathrm{SNe}$ Ia occur preferentially in massive elliptical galaxies (e.g., Taubenberger 2017), which tend to be relatively metal-rich. Therefore, it might be the case that the same mechanism that causes such explosions to produce less ${ }^{56} \mathrm{Ni}$ also affects the formation of the carbon trough.

Carbon was also detected in SN 2011fe (Nugent et al. 2011; Parrent et al. 2012). For SN 2011fe, a subsolar metallicity was inferred from the tomography of Mazzali et al. (2014), as well as from comparisons with its "twin," SN 2011by (which also exhibits a carbon trough). Foley \& Kirshner (2013) concluded that SN 2011fe had a lower luminosity, and Graham et al. (2015) noted that the distance to SN 2011by might be underestimated but that then both these SNe Ia would arise
Table 1

Simulation Properties

\begin{tabular}{cccccc}
\hline \hline Model & $\begin{array}{c}t \\
(\text { days })\end{array}$ & $\begin{array}{c}\lg L \\
\left(L_{\mathrm{bol}} / L_{\odot}\right)\end{array}$ & $\begin{array}{c}v_{\text {inner }} \\
\left(\mathrm{km} \mathrm{s}^{-1}\right)\end{array}$ & $\begin{array}{c}T_{\text {inner }}{ }^{\mathrm{a}} \\
(\mathrm{K})\end{array}$ & $\begin{array}{c}T_{\text {inner }}{ }^{\mathrm{b}} \\
(\mathrm{K})\end{array}$ \\
\hline $05 \mathrm{bl}$ & -6 & 8.520 & 8350 & 9765 & 9639 \\
& -5 & 8.617 & 8100 & 10,136 & 9857 \\
& -3 & 8.745 & 7600 & 10,633 & 10,463 \\
& 4.8 & 8.861 & 6800 & 8931 & 8920 \\
& 12.9 & 8.594 & 3350 & 10,072 & 9916 \\
\hline $11 \mathrm{fe}$ & 3.7 & 7.903 & 13,300 & 10,800 & 10,789 \\
& 5.9 & 8.505 & 12,400 & 12,100 & 12,012 \\
& 9.0 & 9.041 & 11,300 & 14,500 & 13,974 \\
& 12.1 & 9.362 & 10,700 & 14,900 & 14,470 \\
& 16.1 & 9.505 & 9000 & 15,100 & 14,856 \\
& 19.1 & 9.544 & 7850 & 14,700 & 14,604 \\
& 22.4 & 9.505 & 6700 & 14,100 & 13,901 \\
& 28.3 & 9.362 & 4550 & 13,500 & 13,515 \\
\hline
\end{tabular}

Notes.

a Temperature at the inner radius as given in the literature (Hachinger et al. 2009; Mazzali et al. 2014).

b Temperature at the inner radius obtained from the simulations used in this work.

from low-metallicity progenitors. In contrast to this, however, Baron et al. (2015) found that, in the context of delayeddetonation models, the strength of the carbon feature is more easily reproduced using solar rather than subsolar abundances.

Yet another example that might help to elucidate the relationship between the carbon trough and the $\mathrm{Fe}$ content of the ejecta is SN iPTF13asv. This overluminous event exhibited a weak (but persistent) carbon signature, while the early spectra showed an absence of Fe features. In addition, this explosion was UV-bright near maximum, its ejecta was clearly stratified, and it originated from a metal-poor host galaxy (Cao et al. 2016).

Fortunately, this also is amenable to verification. With more detailed models, it should be possible to understand exactly where in the ejecta the presence of iron-group elements makes the carbon feature harder to detect. Furthermore, observationally, it should be possible to determine whether the carbon feature is easier to detect for SNe Ia from low-metallicity progenitors by correlating with the metallicity of environments as measured by, e.g., Anderson et al. (2015).

We thank Melissa L. Graham for meaningful discussions about the carbon feature in SNe Ia and its possible connection with the metallicity of the progenitor star and Stephan Hachinger for providing us with the input parameters used in his tomography work of SN $2005 \mathrm{bl}$.

\section{Appendix Simulation Parameters}

Here we present the set of parameters used in our simulations. We attempted to reproduce the tomography analysis of SN 2011fe as published by Mazzali et al. (2014) and SN 2005bl as published by Hachinger et al. (2009). Specifically, to make our assumptions as similar as possible to those used in previous works, we adopted the downbranch, dilute-blackbody, dilute-lte, and nebular modes to treat line interaction, radiative rates, excitation, and ionization, respectively. The model inputs and the temperature at the inner boundary are given in Table 1, while the abundance 
Table 2

Abundance Stratification

\begin{tabular}{|c|c|c|c|c|c|c|c|c|c|c|c|c|c|}
\hline Model & $v\left(\mathrm{~km} \mathrm{~s}^{-1}\right)$ & $X(\mathrm{C})$ & $X(\mathrm{O})$ & $X(\mathrm{Na})$ & $X(\mathrm{Mg})$ & $X(\mathrm{Al})$ & $X(\mathrm{Si})$ & $X(\mathrm{~S})$ & $X(\mathrm{Ca})$ & $X(\mathrm{Ti})$ & $X(\mathrm{Cr})$ & $X(\mathrm{Fe})_{0}{ }^{\mathrm{a}}$ & $X(\mathrm{Ni})_{0}{ }^{\mathrm{a}}$ \\
\hline \multirow[t]{5}{*}{ 05bl } & $16,000-33,000$ & 0.4184 & 0.5726 & 0.0000 & 0.0030 & 0.0000 & 0.0050 & 0.0010 & 0.0000 & 0.0000 & 0.0000 & 0.0000 & 0.0000 \\
\hline & $8400-16,000$ & 0.0600 & 0.8600 & 0.0060 & 0.0400 & 0.0025 & 0.0200 & 0.0100 & 0.0004 & 0.0004 & 0.0003 & 0.0003 & 0.0000 \\
\hline & $8100-8400$ & 0.0300 & 0.1300 & 0.0030 & 0.0300 & 0.0025 & 0.6800 & 0.1000 & 0.0004 & 0.0100 & 0.0070 & 0.0150 & 0.0000 \\
\hline & $6600-7500$ & 0.0000 & 0.0000 & 0.0000 & 0.0000 & 0.0000 & 0.7100 & 0.0700 & 0.0004 & 0.0550 & 0.0400 & 0.1150 & 0.0100 \\
\hline & $3300-6600$ & 0.0000 & 0.0000 & 0.0000 & 0.0000 & 0.0000 & 0.7700 & 0.0000 & 0.0005 & 0.0167 & 0.0167 & 0.0650 & 0.1300 \\
\hline & $13,500-16,000$ & 0.0310 & 0.7030 & 0.0000 & 0.0300 & 0.0000 & 0.2000 & 0.0300 & 0.0030 & 0.0005 & 0.0005 & 0.0010 & 0.0010 \\
\hline & $12,000-13,500$ & 0.0000 & 0.3510 & 0.0000 & 0.0200 & 0.0000 & 0.4400 & 0.0800 & 0.0030 & 0.0030 & 0.0030 & 0.0600 & 0.0400 \\
\hline & $11,000-12,000$ & 0.0080 & 0.1100 & 0.0000 & 0.0000 & 0.0000 & 0.5630 & 0.1500 & 0.0030 & 0.0050 & 0.0050 & 0.0060 & 0.1500 \\
\hline & $9000-11,000$ & 0.0080 & 0.0900 & 0.0000 & 0.0000 & 0.0000 & 0.4785 & 0.1500 & 0.0030 & 0.0050 & 0.0050 & 0.0005 & 0.2600 \\
\hline & $8500-9000$ & 0.0080 & 0.0900 & 0.0000 & 0.0000 & 0.0000 & 0.1985 & 0.0700 & 0.0030 & 0.0050 & 0.0050 & 0.0005 & 0.6200 \\
\hline & $8000-8500$ & 0.0080 & 0.0200 & 0.0000 & 0.0000 & 0.0000 & 0.2285 & 0.0700 & 0.0030 & 0.0050 & 0.0050 & 0.0005 & 0.6600 \\
\hline
\end{tabular}

Notes.

${ }^{\mathrm{a}}$ For convenience, $\mathrm{Fe}$ and Ni mass fractions are given at $t=0$, before the decay rates are taken into account.

stratification adopted is given in Table 2. For comparison purposes, we have also run the simulations adopting the more physical line interaction mode, macroatom, while keeping all other parameters unchanged. While the differences in the computed spectra are not negligible, they do not affect our main conclusions.

\section{ORCID iDs}

E. Heringer (1) https://orcid.org/0000-0002-1502-9127 M. H. van Kerkwijk (1) https://orcid.org/0000-00025830-8505

S. A. Sim (1) https://orcid.org/0000-0002-9774-1192

W. E. Kerzendorf (i) https://orcid.org/0000-0002-0479-7235

\section{References}

Anderson, J. P., James, P. A., Förster, F., et al. 2015, MNRAS, 448, 732 Ashall, C., Mazzali, P. A., Pian, E., \& James, P. A. 2016, MNRAS, 463, 1891 Baron, E., Hoeflich, P., Friesen, B., et al. 2015, MNRAS, 454, 2549 Benetti, S., Cappellaro, E., Mazzali, P. A., et al. 2005, ApJ, 623, 1011 Blondin, S., Dessart, L., Hillier, D. J., \& Khokhlov, A. M. 2013, MNRAS, 429, 2127

Blondin, S., Matheson, T., Kirshner, R. P., et al. 2012, AJ, 143, 126

Botyánszki, J., \& Kasen, D. 2017, arXiv:1704.06275

Branch, D., Chau Dang, L., \& Baron, E. 2009, PASP, 121, 238

Branch, D., Dang, L. C., Hall, N., et al. 2006, PASP, 118, 560

Branch, D., Jeffery, D. J., Parrent, J., et al. 2008, PASP, 120, 135

Cao, Y., Johansson, J., Nugent, P. E., et al. 2016, ApJ, 823, 147

Chomiuk, L., Soderberg, A. M., Chevalier, R. A., et al. 2016, ApJ, 821, 119

Dhawan, S., Leibundgut, B., Spyromilio, J., \& Blondin, S. 2017, A\&A, 602, A118

Dong, S., Katz, B., Kushnir, D., \& Prieto, J. L. 2015, MNRAS, 454, L61

Doull, B. A., \& Baron, E. 2011, PASP, 123, 765

Filippenko, A. V. 1997, ARA\&A, 35, 309

Folatelli, G., Phillips, M. M., Morrell, N., et al. 2012, ApJ, 745, 74

Foley, R. J., \& Kirshner, R. P. 2013, ApJL, 769, L1

Graham, M. L., Foley, R. J., Zheng, W., et al. 2015, MNRAS, 446, 2073

Graur, O., Bianco, F. B., Modjaz, M., et al. 2017, ApJ, 837, 121

Hachinger, S., Mazzali, P. A., Tanaka, M., Hillebrandt, W., \& Benetti, S. 2008, MNRAS, 389, 1087
Hachinger, S., Mazzali, P. A., Taubenberger, S., Pakmor, R., \& Hillebrandt, W. 2009, MNRAS, 399, 1238

Iwamoto, K., Brachwitz, F., Nomoto, K., et al. 1999, ApJS, 125, 439

Kerzendorf, W. E., \& Sim, S. A. 2014, MNRAS, 440, 387

Kromer, M., Sim, S. A., Fink, M., et al. 2010, ApJ, 719, 1067

Lentz, E. J., Baron, E., Branch, D., Hauschildt, P. H., \& Nugent, P. E. 2000, ApJ, 530, 966

Liu, Y.-Q., Modjaz, M., Bianco, F. B., \& Graur, O. 2016, ApJ, 827, 90

Livne, E. 1990, ApJL, 354, L53

Mazzali, P. A. 2000, A\&A, 363, 705

Mazzali, P. A., Chugai, N., Turatto, M., et al. 1997, MNRAS, 284, 151

Mazzali, P. A., Sauer, D. N., Pastorello, A., Benetti, S., \& Hillebrandt, W. 2008, MNRAS, 386, 1897

Mazzali, P. A., Sullivan, M., Filippenko, A. V., et al. 2015, MNRAS, 450, 2631

Mazzali, P. A., Sullivan, M., Hachinger, S., et al. 2014, MNRAS, 439, 1959

McClelland, C. M., Garnavich, P. M., Milne, P. A., Shappee, B. J., \& Pogge, R. W. 2013, ApJ, 767, 119

Milne, P. A., \& Brown, P. J. 2012, arXiv:1201.1279

Nomoto, K., Thielemann, F.-K., \& Yokoi, K. 1984, ApJ, 286, 644

Nugent, P., Phillips, M., Baron, E., Branch, D., \& Hauschildt, P. 1995, ApJL, 455, L147

Nugent, P. E., Sullivan, M., Cenko, S. B., et al. 2011, Natur, 480, 344

Pakmor, R., Kromer, M., Röpke, F. K., et al. 2010, Natur, 463, 61

Pakmor, R., Kromer, M., Taubenberger, S., et al. 2012, ApJL, 747, L10

Parrent, J. T., Howell, D. A., Friesen, B., et al. 2012, ApJL, 752, L26

Pastorello, A., Mazzali, P. A., Pignata, G., et al. 2007, MNRAS, 377, 1531

Piro, A. L., Thompson, T. A., \& Kochanek, C. S. 2014, MNRAS, 438, 3456

Sasdelli, M., Hillebrandt, W., Kromer, M., et al. 2017, MNRAS, 466, 3784

Sasdelli, M., Ishida, E. E. O., Vilalta, R., et al. 2016, MNRAS, 461, 2044

Sauer, D. N., Mazzali, P. A., Blondin, S., et al. 2008, MNRAS, 391, 1605

Shen, K. J., Kasen, D., Miles, B. J., \& Townsley, D. M. 2017, arXiv:1706. 01898

Silverman, J. M., \& Filippenko, A. V. 2012, MNRAS, 425, 1917

Silverman, J. M., Foley, R. J., Filippenko, A. V., et al. 2012a, MNRAS, 425,1789

Silverman, J. M., Kong, J. J., \& Filippenko, A. V. 2012b, MNRAS, 425, 1819

Stehle, M., Mazzali, P. A., Benetti, S., \& Hillebrandt, W. 2005, MNRAS, 360,1231

Taubenberger, S. 2017, in Handbook of Supernovae, ed. A. Alsabti \& P. Murdin (New York: Springer), 50

Taubenberger, S., Hachinger, S., Pignata, G., et al. 2008, MNRAS, 385, 75

Thomas, R. C., Aldering, G., Antilogus, P., et al. 2011, ApJ, 743, 27

Walker, E. S., Hachinger, S., Mazzali, P. A., et al. 2012, MNRAS, 427, 103

Yaron, O., \& Gal-Yam, A. 2012, PASP, 124, 668 